\title{
Advances in embryo selection methods
}

\section{Anna Ajduk ${ }^{1}$ and Magdalena Zernicka-Goetz ${ }^{2 *}$}

Addresses: ${ }^{1}$ University of Warsaw, Department of Embryology, Miecznikowa 1, 02-096 Warsaw, Poland; ${ }^{2}$ University of Cambridge, The Wellcome Trust/Cancer Research Gurdon Institute, Tennis Court Road, CB2 1QN, Cambridge, UK

*Corresponding author: Magdalena Zernicka-Goetz (m.zernicka-goetz@gurdon.cam.ac.uk)

Fl000 Biology Reports 2012, 4:II (doi:I0.34I0/B4-II)

This is an open-access article distributed under the terms of the Creative Commons Attribution-Non Commercial License (http://creativecommons.org/licenses/by-nc/3.0/legalcode), which permits unrestricted use, distribution, and reproduction in any medium, provided the original work is properly cited. You may not use this work for commercial purposes.

The electronic version of this article is the complete one and can be found at: http://fl $000 . c o m / r e p o r t s / b / 4 / I I$

\begin{abstract}
Despite many recent advances in the field of reproductive biology and medicine, the efficiency of in vitro fertilization procedures remains relatively low. There is a need for a reliable and non-invasive method of embryo selection to ensure that only embryos with the highest developmental potential are chosen for transfer to mothers-to-be. Here, we compare various methods currently used for assessing embryonic viability, such as examination of embryonic morphology, quality of the genetic material, or metabolism. Additionally, we discuss novel procedures for embryonic assessment based on advanced time-lapse imaging techniques, which show great promise and may lead to increased in vitro fertilization efficiencies.
\end{abstract}

\section{Introduction}

Since 1978, when the first baby conceived in vitro was born, in vitro fertilization (IVF) has become a major method for treating infertility. Although IVF procedures have been greatly improved over the years, their efficiency, as defined by live birth rate, is still only about $30-40 \%$. Consequently, many couples have to undergo treatment several times before they succeed and this increases emotional and financial costs, as well as additional health risks for women. The efficiency of IVF can be increased by transferring multiple embryos in a single cycle, but this may lead to multiple-pregnancies, and consequently serious health complications for mothers and babies. There is, therefore, a strong need for single embryo transfers, and so the development of reliable methods of selecting embryos is crucial $[1,2]$. Here, we provide a short review of current techniques for embryo assessment, with a focus on new ways of distinguishing high-quality embryos based on recent advances in time-lapse imaging technology.

\section{Static assessment of embryo morphology by light microscopy}

Visual assessment of embryo morphology is the most traditional and popular method of embryo selection. Several parameters can be assessed at different developmental stages, providing valuable information about the quality of embryos [3]. Embryos can be graded according to the morphology of their pronuclei on day 1 after fertilization [4-6], the number and shape of blastomeres and degree of fragmentation on day 2 or 3 [7-9], or by the morphology of the blastocyst at day 5 or 6 [10-12]. Depending on the procedure, embryos are evaluated at one or several developmental stages.

Although morphological assessment is inexpensive and easy to implement in a clinical environment, it has its drawbacks. Firstly, the visual grading of embryos is subjective and requires significant expertise. Moreover, even when such expertise is available, the technique is not always accurate: low-graded embryos often prove to have high developmental potential and can develop to term. Therefore, there has been a drive to develop an alternative method of embryo evaluation that provides more detailed information about the developmental status of the embryo and, most importantly, is quantitative and objective.

\section{Preimplantation genetic diagnosis and screening}

Preimplantation genetic diagnosis has been developed to detect inherited genetic disorders. It uses polymerase chain reaction (PCR)-based techniques to diagnose specific 
genetic mutations [13] or fluorescence in situ hybridization (FISH) to diagnose chromosomal abnormalities or to sex the embryos for patients carrying X-chromosome-linked diseases [14-16]. Preimplantation genetic diagnosis can be applied to embryos at different stages: zygotes (polar body biopsy), cleavage stage embryos (blastomere biopsy) or blastocysts (trophectoderm biopsy).

An alternative approach is preimplantation genetic screening, which was developed to improve IVF outcomes in mothers of advanced age, those presenting with repeated miscarriages or implantation failure, or in cases of severe male factor infertility. The classic form of preimplantation genetic screening, involving FISH assessment of a limited number of chromosomes in one blastomere of a cleavage stage embryo, has been shown to be ineffective and has been gradually replaced by technologies based on comparative genomic hybridization (CGH) or single nucleotide polymorphism (SNP) arrays $[17,18]$. Both of these approaches allow the whole embryonic genome to be analysed and, as for preimplantation genetic diagnosis, used at different embryonic stages.

\section{Metabolomics and proteomics in screening}

Another method of embryo selection is based on the analysis of embryo metabolism. Changes in pyruvate or glucose concentration in the culture medium can reflect the energy metabolism of the embryo, although their usefulness as a tool to predict the embryo's quality is not clear [19-23]. On the other hand, oxygen consumption [24-27] and amino-acid turnover [28-30] have proved to be more reliable indicators of an embryo's viability. Recently, the analysis of single metabolites has been gradually replaced by a broader approach: metabolomic or protein secrotome profiling [31], which can provide a complete picture of an embryo's metabolism and gene expression patterns. Although many reports have shown a correlation between metabolic status of the embryo and its viability, establishing its potential value for the IVF clinics, for the moment these techniques remain difficult to implement in a clinical environment. This is because metabolomic or secreted protein analyses involve spectroscopic/spectrometric and chromatographic techniques, which currently require expensive equipment and highly skilled staff [31].

\section{Dynamic assessment of embryonic development by time-lapse imaging}

Due to recent advances in non-invasive time-lapse imaging established in mouse embryos [32], we can now follow the dynamics of embryo divisions and other fertilizationtriggered events and correlate them with the developmental potential of the embryos. The last two years have seen two studies employing this technique in a very different way. A team at Stanford University has shown that the timing and synchrony of the first two embryonic divisions are predictive of developmental potential of human embryos (Figure 1) [33]. The authors reported that embryos with a very long first cytokinesis, with a prolonged or an atypically short interval between first and second division or with highly asynchronous divisions of two-cell blastomeres, fail to reach the blastocyst stage. This accords with previous observations, in which timely pronuclear formation and subsequent first cleavage correlated with higher quality of human embryos [34-36]. A completely different approach has been developed by our team at University of Cambridge working collaboratively with teams at Oxford and Cardiff Universities (Figure 2) [37].

Figure I. Novel methods of predicting mammalian embryo viability involving an advanced time-lapse imaging

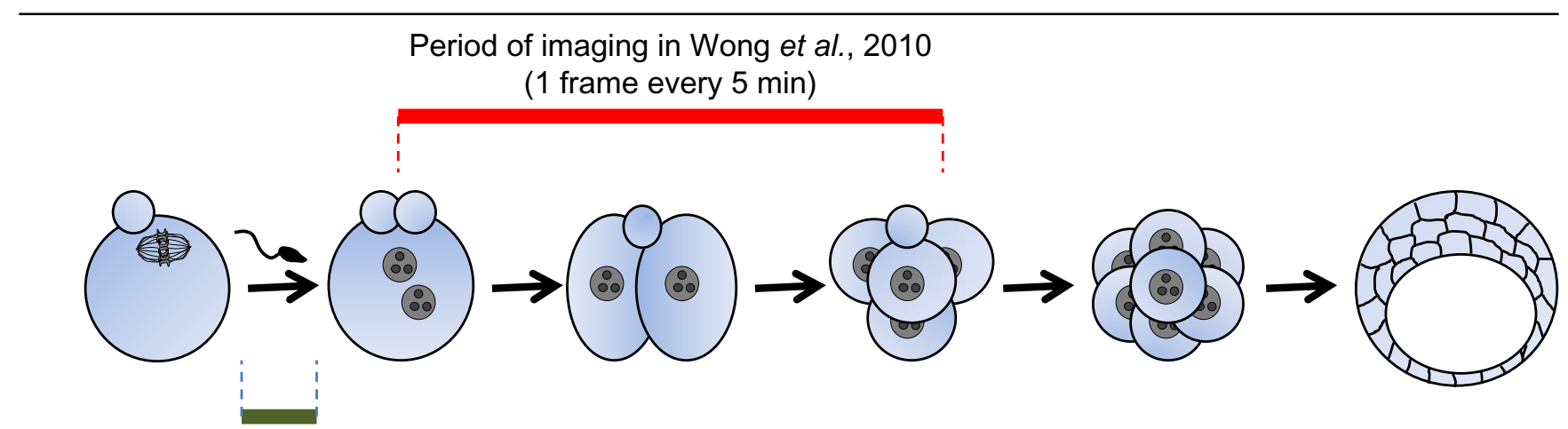

Period of imaging in Ajduk et al., 2011

(1 frame every 10s)

The method described by Wong et al. involves time-lapse imaging of embryos every $5 \mathrm{~min}$ for several hours (from I- to 4-cell stage) [33]. In comparison, the method established by Ajduk et al. requires a much shorter period of recording ( 2.5 hours for mouse embryos) but with images taken every 10 seconds [37]. 
Figure 2. Analysis of fertilization-triggered cytoplasmic movements in mouse zygotes

\section{Time-lapse imaging}

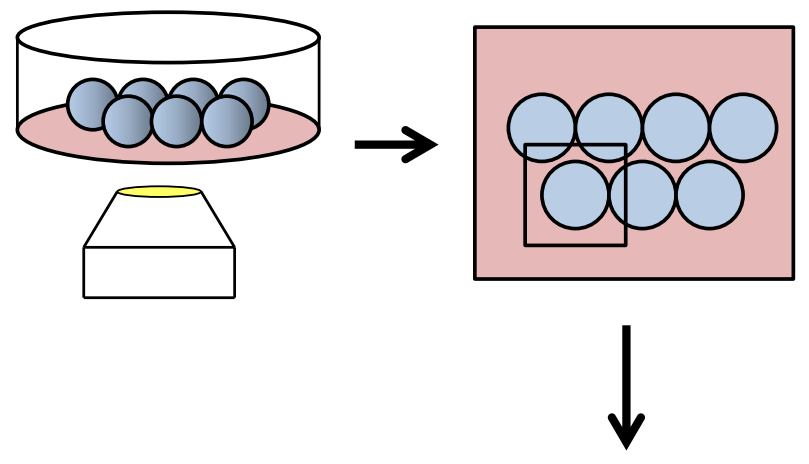

Particle Image Velocimetry analysis

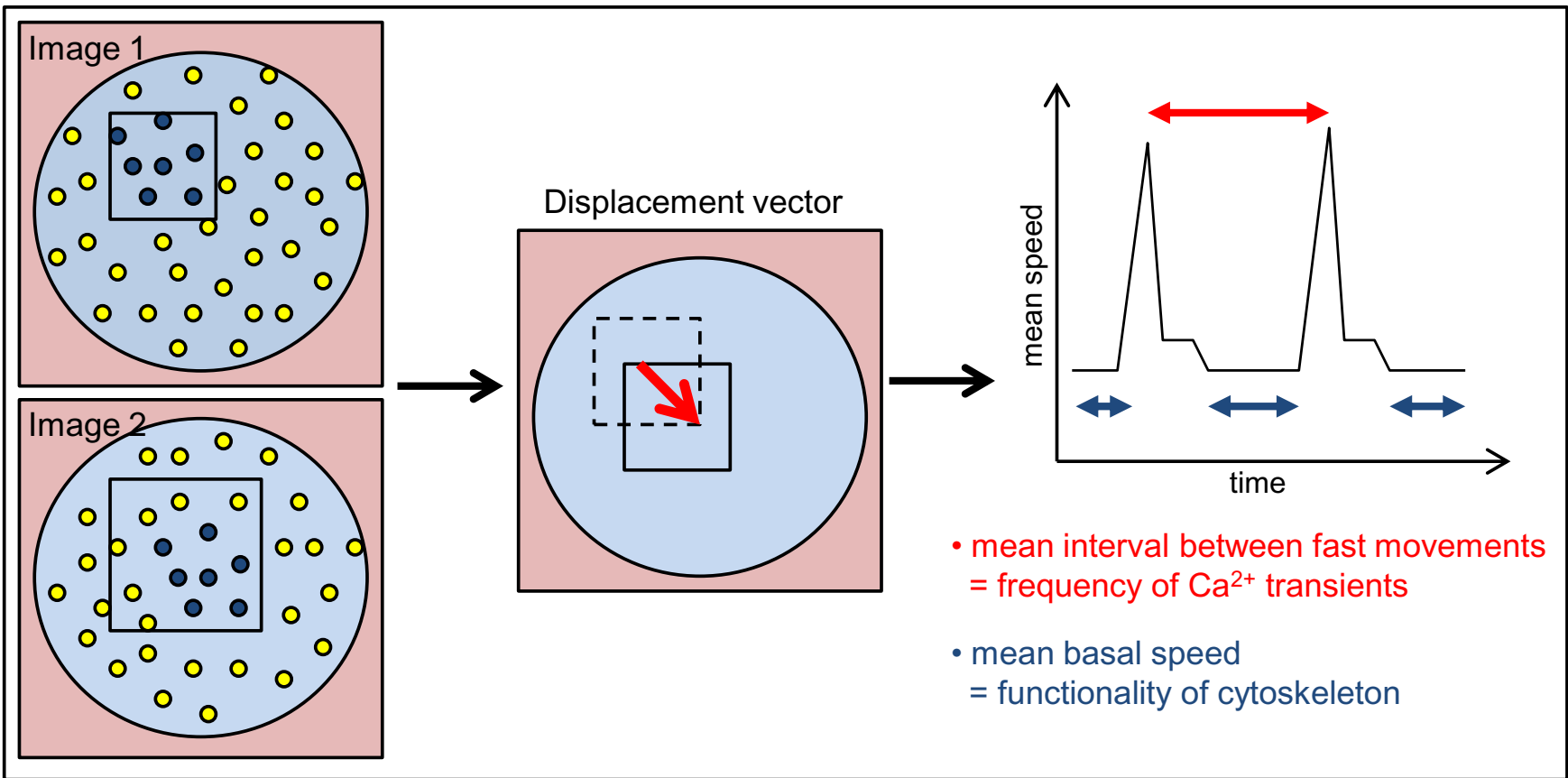

Mouse eggs are subjected to time-lapse imaging ( 1 frame every 10 seconds for 2.5 hours) immediately after fertilization [37]. Acquired images are analysed by the particle image velocimetry method that follows patterns of contrasts between subsequent images and calculates how they move. The sum of all displacement vectors calculated for the zygote in a given time-point (i.e. mean cytoplasmic speed) is plotted over time. The graph shows when fast cytoplasmic movements occurred in the embryo. Mean interval between the fast movements (in red) and mean speed in periods inbetween the fast movements (mean basal speed, in blue) are indicative of developmental potential of the embryo.

In this work, we showed that fertilization of mouse eggs triggers abrupt, repetitive cytoplasmic movements that correlated with $\mathrm{Ca}^{2+}$ oscillations (also triggered by sperm) and depended on the functionality of the cytoskeleton. Moreover, the cytoplasmic flows were predictive of the developmental potential of the embryos. Embryos that showed very frequent increases in cytoplasmic movements (indicating very frequent $\mathrm{Ca}^{2+}$ oscillations) and low cytoplasmic speeds in the intervening periods (reflecting low quality of the actin cytoskeleton) were three-fold less successful in developing to pups than embryos displaying average values of these parameters. Although both methods still have to be tested in a clinical environment and subjected to randomised controlled trials to demonstrate improved live birth outcomes, they offer great hope for more reliable assessment of embryonic quality. 


\section{Conclusion}

Research carried out over the past three decades has provided a broad repertoire of possible improved embryo selection methods. Some of them, such as evaluation of pronuclear morphology, preimplantation genetic diagnosis and preimplantation genetic screening, focus on the nuclear component of the embryo, looking for potential chromosomal abnormalities. Others (e.g. analysis of metabolites, secreted proteins or cytoplasmic flows) concentrate on the cytoplasmic component, analysing the quality of embryo metabolism, the cytoskeleton or $\mathrm{Ca}^{2+}$ homeostasis. We believe that the ultimate selection method should combine these approaches. This can be achieved for instance by following the timing of embryonic cell divisions, as their duration and synchrony can be affected by improper chromosome segregation, cytoskeletal properties and energy levels. An alternative approach could combine preimplantation genetic screening with the analysis of embryo metabolism or cytoplasmic movements. Combination of genetic testing of the polar body and examination of the cytoplasmic flows is especially promising, as it would provide information about embryo quality within several hours after fertilization, and, therefore, significantly quicken the selection process (Figure 1). Indeed, recent publications suggest that a shorter period between in vitro fertilization and embryo transfer may be beneficial, as prolonged in vitro culture of embryos alters their epigenetic modifications and gene expression $[38,39]$. Through a combination of these new methods, it is reasonable to expect that embryo selection for IVF will become much more reliable in the coming years.

\section{Abbreviations}

FISH, fluorescence in situ hybridization; IVF, in vitro fertilization; PCR, polymerase chain reaction.

\section{Competing interests}

The method of assessing embryo developmental potential based on analysis of the cytoplasmic movements is the subject of a patent application filed in the U.S. by Magdalena Zernicka-Goetz and Anna Ajduk (US application no. 61/503827).

\section{References}

I. Gerris J: Single-embryo transfer versus multiple-embryo transfer. Reprod Biomed Online 2009, I8(Suppl 2):63-70.

FI000 Factor 6

Evaluated by Magdalena Zernicka-Goetz and Anna Ajduk 17 May 2012

2. Gerris JM: Single embryo transfer and IVF/ICSI outcome: a balanced appraisal. Hum Reprod Update 2005, I I: I05-2I.

3. Ebner T, Moser M, Sommergruber M, Tews G: Selection based on morphological assessment of oocytes and embryos at different stages of preimplantation development: a review. Hum Reprod Update 2003, 9:25I-62.

FI000 Factor 6

Evaluated by Magdalena Zernicka-Goetz and Anna Ajduk 17 May 2012

4. Tesarik J, Greco E: The probability of abnormal preimplantation development can be predicted by a single static observation on pronuclear stage morphology. Hum Reprod 1999, 14:1318-23.

FI000 Factor 6

Evaluated by Magdalena Zernicka-Goetz and Anna Ajduk 17 May 2012

5. Scott LA, Smith S: The successful use of pronuclear embryo transfers the day following oocyte retrieval. Hum Reprod 1998, 13:1003-13.

6. Montag M, van der Ven $\mathrm{H}$ : Evaluation of pronuclear morphology as the only selection criterion for further embryo culture and transfer: results of a prospective multicentre study. Hum Reprod 2001, 16:2384-9.

7. Van Royen E, Mangelschots K, De Neubourg D, Valkenburg M, Van de Meerssche M, Ryckaert G, Eestermans W, Gerris J: Characterization of a top quality embryo, a step towards single-embryo transfer. Hum Reprod 1999, 14:2345-9.

8. Hardarson T, Hanson C, Sjogren A, Lundin K: Human embryos with unevenly sized blastomeres have lower pregnancy and implantation rates: indications for aneuploidy and multinucleation. Hum Reprod 200I, 16:313-8.

9. Ziebe S, Petersen K, Lindenberg S, Andersen AG, Gabrielsen A, Andersen AN: Embryo morphology or cleavage stage: how to select the best embryos for transfer after in-vitro fertilization. Hum Reprod 1997, I 2:1545-9.

FI000 Factor 6

Evaluated by Magdalena Zernicka-Goetz and Anna Ajduk 17 May 2012

10. Gardner DK, Lane M, Stevens J, Schlenker T, Schoolcraft WB: Blastocyst score affects implantation and pregnancy outcome: towards a single blastocyst transfer. Fertil Steril 2000, 73:1155-8.

FI000 Factor 6

Evaluated by Magdalena Zernicka-Goetz and Anna Ajduk 17 May 2012

II. Richter KS, Harris DC, Daneshmand ST, Shapiro BS: Quantitative grading of a human blastocyst: optimal inner cell mass size and shape. Fertil Steril 200I, 76:1 157-67.

12. Balaban B, Urman B, Sertac A, Alatas C, Aksoy S, Mercan R: Blastocyst quality affects the success of blastocyst-stage embryo transfer. Fertil Steril 2000, 74:282-7.

13. Spits C, Sermon K: PGD for monogenic disorders: aspects of molecular biology. Prenat Diagn 2009, 29:50-6.

FI000 Factor 6

Evaluated by Magdalena Zernicka-Goetz and Anna Ajduk 17 May 2012

14. Griffin DK, Handyside AH, Harper JC, Wilton LJ, Atkinson G, Soussis I, Wells D, Kontogianni E, Tarin J, Geber S, Asangla AO, Winston RM, Delhanty JDA: Clinical experience with preimplantation diagnosis of sex by dual fluorescent in situ hybridization. J Assist Reprod Genet 1994, I I:I32-43.

FI000 Factor 6

Evaluated by Magdalena Zernicka-Goetz and Anna Ajduk 17 May 2012

15. Munne S, Weier HU, Stein J, Grifo J, Cohen J: A fast and efficient method for simultaneous $X$ and $Y$ in situ hybridization of human blastomeres. J Assist Reprod Genet 1993, 10:82-90.

FI000 Factor 6

Evaluated by Magdalena Zernicka-Goetz and Anna Ajduk 17 May 2012 
16. Harper JC, Coonen E, Ramaekers FC, Delhanty JD, Handyside AH, Winston RM, Hopman AH: Identification of the sex of human preimplantation embryos in two hours using an improved spreading method and fluorescent in-situ hybridization (FISH) using directly labelled probes. Hum Reprod 1994, 9:72 I-4.

FI000 Factor 6

Evaluated by Magdalena Zernicka-Goetz and Anna Ajduk 17 May 2012

17. Harper JC, Harton G: The use of arrays in preimplantation genetic diagnosis and screening. Fertil Steril 2010, 94:1 173-7.

FI000 Factor 6

Evaluated by Magdalena Zernicka-Goetz and Anna Ajduk 17 May 2012

18. Harper JC, Sengupta SB: Preimplantation genetic diagnosis: State of the ART 20II. Hum Genet 20II, I3 I:175-86.

FI000 Factor 6

Evaluated by Magdalena Zernicka-Goetz and Anna Ajduk 17 May 2012

19. Gardner DK, Lane M, Stevens J, Schoolcraft WB: Noninvasive assessment of human embryo nutrient consumption as a measure of developmental potential. Fertil Steril 200 I, 76: I I 75-80.

20. Conaghan J, Handyside AH, Winston RM, Leese HJ: Effects of pyruvate and glucose on the development of human preimplantation embryos in vitro. J Reprod Fertil 1993, 99:87-95.

21. Hardy K, Hooper MA, Handyside AH, Rutherford AJ, Winston RM, Leese HJ: Non-invasive measurement of glucose and pyruvate uptake by individual human oocytes and preimplantation embryos. Hum Reprod 1989, 4:188-91.

22. Gott AL, Hardy K, Winston RM, Leese HJ: Non-invasive measurement of pyruvate and glucose uptake and lactate production by single human preimplantation embryos. Hum Reprod 1990, 5:104-8.

23. Jones GM, Trounson AO, Vella PJ, Thouas GA, Lolatgis N, Wood C: Glucose metabolism of human morula and blastocyst-stage embryos and its relationship to viability after transfer. Reprod Biomed Online 2001, 3:124-32.

24. Magnusson C, Hillensjo T, Hamberger L, Nilsson L: Oxygen consumption by human oocytes and blastocysts grown in vitro. Hum Reprod 1986, I:183-4.

25. Lopes AS, Larsen LH, Ramsing N, Lovendahl P, Raty M, Peippo J, Greve $\mathrm{T}$, Callesen $\mathrm{H}$ : Respiration rates of individual bovine in vitro-produced embryos measured with a novel, non-invasive and highly sensitive microsensor system. Reproduction 2005, 130:669-79.

26. Lopes AS, Madsen SE, Ramsing NB, Lovendahl P, Greve T, Callesen H: Investigation of respiration of individual bovine embryos produced in vivo and in vitro and correlation with viability following transfer. Hum Reprod 2007, 22:558-66.

FI000 Factor 6

Evaluated by Magdalena Zernicka-Goetz and Anna Ajduk 17 May 2012

27. Shiku H, Shiraishi T, Ohya H, Matsue T, Abe H, Hoshi H, Kobayashi M: Oxygen consumption of single bovine embryos probed by scanning electrochemical microscopy. Anal Chem 2001, 73:3751-8.

28. Seli E, Botros L, Sakkas D, Burns DH: Noninvasive metabolomic profiling of embryo culture media using proton nuclear magnetic resonance correlates with reproductive potential of embryos in women undergoing in vitro fertilization. Fertil Steril 2008, 90:2183-9.

FI000 Factor 6

Evaluated by Magdalena Zernicka-Goetz and Anna Ajduk 17 May 2012

29. Houghton FD, Hawkhead JA, Humpherson PG, Hogg JE, Balen AH, Rutherford $A$, , Leese $H$ J: Non-invasive amino acid turnover predicts human embryo developmental capacity. Hum Reprod 2002, I7:999-1005.

30. Brison DR, Houghton FD, Falconer D, Roberts SA, Hawkhead J, Humpherson PG, Lieberman BA, Leese HJ: Identification of viable embryos in IVF by non-invasive measurement of amino acid turnover. Hum Reprod 2004, 19:2319-24.

31. Nel-Themaat L, Nagy ZP: A review of the promises and pitfalls of oocyte and embryo metabolomics. Placenta 201I, 32(Suppl 3): S257-63.

FI000 Factor 6

Evaluated by Magdalena Zernicka-Goetz and Anna Ajduk 17 May 2012

32. Bischoff M, Parfitt DE, Zernicka-Goetz M: Formation of the embryonic-abembryonic axis of the mouse blastocyst: relationships between orientation of early cleavage divisions and pattern of symmetric/asymmetric divisions. Development 2008, 135:953-62.

33. Wong CC, Loewke KE, Bossert NL, Behr B, De Jonge CJ, Baer TM, Reijo Pera RA: Non-invasive imaging of human embryos before embryonic genome activation predicts development to the blastocyst stage. Nat Biotechnol 2010, 28: III5-2I.

FI000 Factor 9

Evaluated by Jon D Hennebold and Marina C Peluffo 19 Oct 2010, Magdalena Zernicka-Goetz and Anna Ajduk 17 May 2012

34. Fenwick J, Platteau P, Murdoch AP, Herbert M: Time from insemination to first cleavage predicts developmental competence of human preimplantation embryos in vitro. Hum Reprod 2002, 17:407-12.

FI000 Factor 6

Evaluated by Magdalena Zernicka-Goetz and Anna Ajduk 17 May 2012

35. Lundin K, Bergh C, Hardarson T: Early embryo cleavage is a strong indicator of embryo quality in human IVF. Hum Reprod 200I, 16:2652-7.

FI000 Factor 6

Evaluated by Magdalena Zernicka-Goetz and Anna Ajduk 17 May 2012

36. Lemmen JG, Agerholm I, Ziebe S: Kinetic markers of human embryo quality using time-lapse recordings of IVF/ICSIfertilized oocytes. Reprod Biomed Online 2008, 17:385-91.

FI000 Factor 6

Evaluated by Magdalena Zernicka-Goetz and Anna Ajduk 17 May 2012

37. Ajduk A, llozue T, Windsor S, Yu Y, Seres KB, Bomphrey RJ, Tom BD, Swann K, Thomas A, Graham C, Zernicka-Goetz M: Rhythmic actomyosin-driven contractions induced by sperm entry predict mammalian embryo viability. Nat Commun 201I, 2:4I7.

FI000 Factor 6

Keith Jones 25 Aug 2011

38. Rinaudo PF, Giritharan G, Talbi S, Dobson AT, Schultz RM: Effects of oxygen tension on gene expression in preimplantation mouse embryos. Fertil Steril 2006, 86: 1252-65.

FI000 Factor 6

Evaluated by Magdalena Zernicka-Goetz and Anna Ajduk 17 May 2012

39. Market-Velker BA, Fernandes AD, Mann MR: Side-by-side comparison of five commercial media systems in a mouse model: suboptimal in vitro culture interferes with imprint maintenance. Biol Reprod 83:938-50.

FI000 Factor 6

Evaluated by Magdalena Zernicka-Goetz and Anna Ajduk 17 May 2012 